\title{
Case Report \\ Open Reduction and Internal Fixation of a Proximal Femoral Shaft Fracture in a Patient with Bilateral Congenital Hip Disease
}

\author{
Stefania Kanata (iD) and Antonios Anastasiadis \\ 6th Orthopaedic Department, KAT General Hospital of Athens, Nikis 2, Kifissia, Athens 145-61, Greece \\ Correspondence should be addressed to Stefania Kanata; steka90@hotmail.com
}

Received 9 September 2017; Accepted 3 January 2018; Published 31 January 2018

Academic Editor: Werner Kolb

Copyright (c) 2018 Stefania Kanata and Antonios Anastasiadis. This is an open access article distributed under the Creative Commons Attribution License, which permits unrestricted use, distribution, and reproduction in any medium, provided the original work is properly cited.

\begin{abstract}
We present a rare case of a femoral shaft fracture in a 74-year-old woman with a preexisting untreated bilateral congenital hip dislocation and with concomitant leg length discrepancy. Because of the marked deformity of the upper femur, a derivative of the congenital hip disease, an open reduction and internal fixation was performed, with the use of an anatomic femur plate originally designed for the treatment of periprosthetic fractures. The patient was treated successfully and returned to her previous status of activity. The management of a femoral fracture in the presence of ipsilateral hip disease is a challenging issue. The surgical treatment choice in such cases has to be individualized, taking into consideration several anatomic and medical parameters.
\end{abstract}

\section{Introduction}

Congenital hip disease (CHD) is relatively common in Greek people [1]. The incidence of untreated cases has been effectively reduced, due to screening methods and early diagnosis. However, still there is sporadic presence of neglected CHD cases, particularly in elders. The occurrence of a femoral shaft fracture in the presence of CHD is extremely rare. Therefore, there are no treatment guidelines for such cases. Intramedullary nailing, the method of choice for the treatment of most femoral shaft fractures, cannot be easily performed in patients with concurrent ipsilateral $\mathrm{CHD}$, especially in those with the severe type of it.

We present here a case of a femoral shaft fracture in a female patient with untreated bilateral congenital hip dislocation. A good final outcome was achieved with internal fixation of the fracture using a periprosthetic fracture plate.

\section{Case Presentation}

A 74-year-old woman was admitted to the emergency department of our hospital after a closed injury of her left femur due to a fall. Plain X-rays showed a spiral shaft fracture of the proximal third of the femur. The radiographs of the pelvis and the patient's history as well revealed that she was also suffering from bilateral congenital hip disease (Figures 1 and 2). The form of dysplasia of the left hip was type B2-low dislocation according to Hartofilakidis classification or type III according to Crowe classification [2-4]. The right hip lesion was more severe, classified as type $\mathrm{C} 2$-high dislocation or type IV according to Hartofilakidis and Crowe classifications, respectively [2-4]. There was a leg length discrepancy of $3 \mathrm{~cm}$, with the injured leg being the longer one. Before the fracture, the left hip was almost immovable, ankylosed in fixed external rotation. The right hip was free of pain with functional range of movement. The patient had never been treated for the $\mathrm{CHD}$, and she was walking with the use of a cane. She was also able to do the usual activities of daily living without significant difficulty. According to her medical history, she was a smoker and was receiving medication for arterial hypertension and hypothyroidism.

Taking into consideration the "personality" of the fracture, the patient's needs, and the surgical options, we performed an open reduction and internal fixation (ORIF) using the NCB periprosthetic femur polyaxial locking plate system (Zimmer 


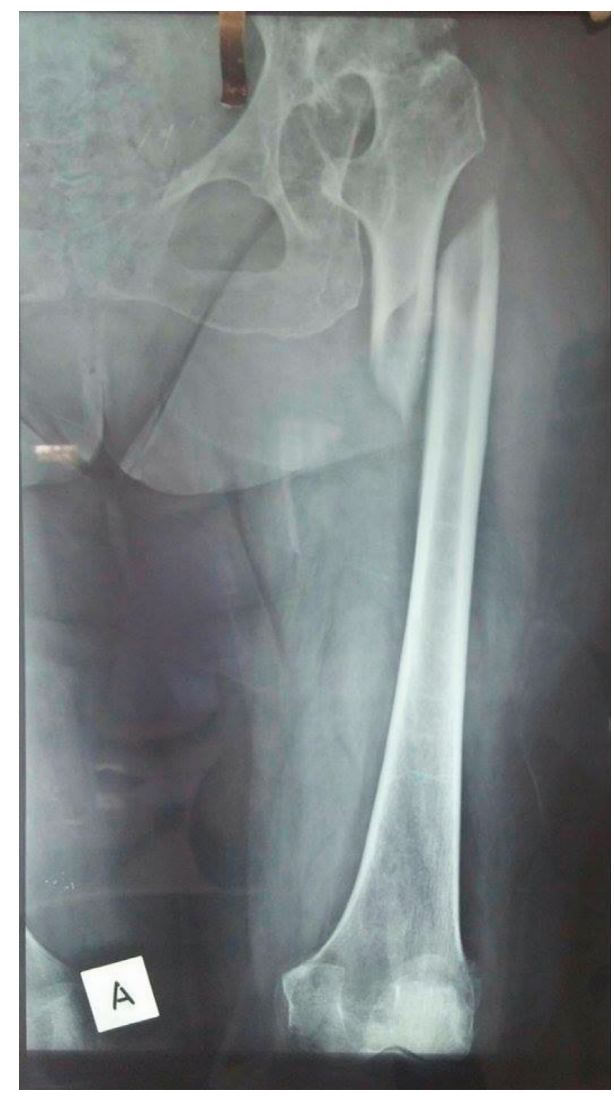

Figure 1: Initial posttraumatic anteroposterior radiograph of the left femur. Congenital hip disease and spiral shaft fracture of the proximal third of the femur are revealed.

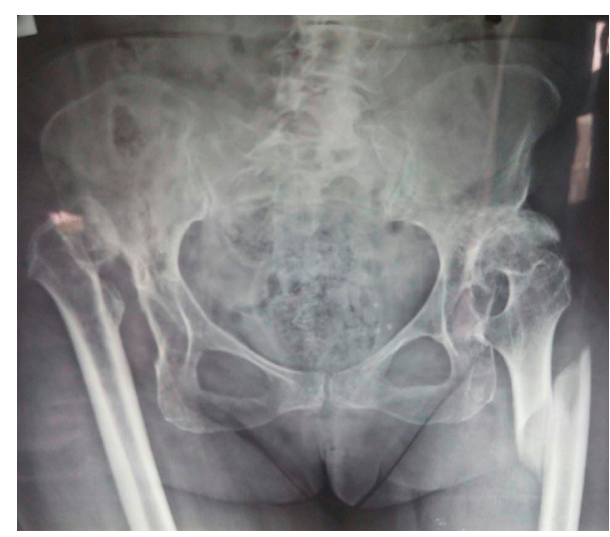

FIGURe 2: Pelvis anteroposterior radiograph showing the neglected hip dislocation of both hips.

Biomet, Indiana, USA). The operation was performed two days after the fracture, under spinal anaesthesia. The patient was placed in the right lateral decubitus position, and the middle and proximal thirds of the femur were accessed through a lateral approach. The subtrochanteric area of the femur was exposed with anterior retraction of vastus lateralis after an L-shaped detachment of its origin. The fracture was anatomically reduced, and two lag screws were placed initially. Then, the NCB periprosthetic femur plate was placed as

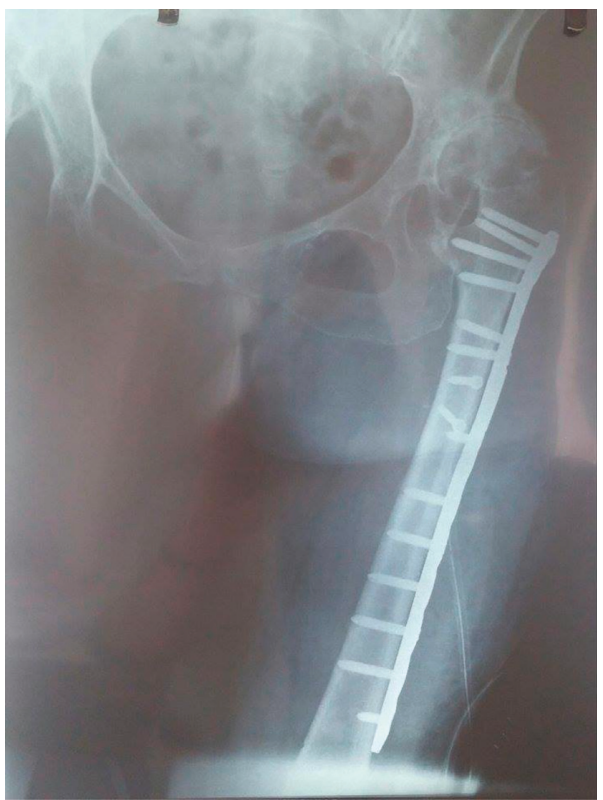

FIgURE 3: Anteroposterior radiograph of the left femur after ORIF (POD 1).

a neutralization plate (Figure 3). Image intensifier was used for the confirmation of the optimal position of the plate. The patient received the standard scheme of perioperative chemoprophylaxis with teicoplanin as well as pre- and postoperative thromboprophylaxis with the use of bemiparin sodium. Intraoperatively, she was transfused with two units of concentrated red blood cells.

The patient was mobilized on the first postoperative day (POD), and she stood upright using crutches, without weight bearing on the fourth POD. The postoperative period was uneventful, and she was discharged on the 6th POD. Because of the leg length discrepancy, she was protected from weight bearing for six weeks when she was reviewed in our outpatient clinic. At that time, the patient was free of pain, and the subsequent $\mathrm{X}$-ray images showed signs of fracture healing (Figures 4 and 5). Following that, she was encouraged to start walking with partial weight bearing. The patient is being followed up for ten months now, and she has fully returned to her previous walking status and activities of daily living.

\section{Discussion}

The combination of a femoral fracture in the presence of preexisting hip pathology is extremely rare. Most of the published reports refer to the cases of either the upper femur or femoral shaft fracture under an arthrodesed hip [5-10]. To the best of our knowledge, there is only one case in the literature concerning the femoral fracture in a preexisting CHD [11].

In our case, it was a noncomminuted spiral fracture of the femoral shaft, caused by rotational forces after a simple fall. The gold standard for those fractures is closed intramedullary nailing (IMN) [12]. However, in patients with $\mathrm{CHD}$, there are alterations in anatomy, increased anteversion (varying from 1 to 80 degrees), increased neck-shaft angle, and hip ankylosis, which make IMN a method with 


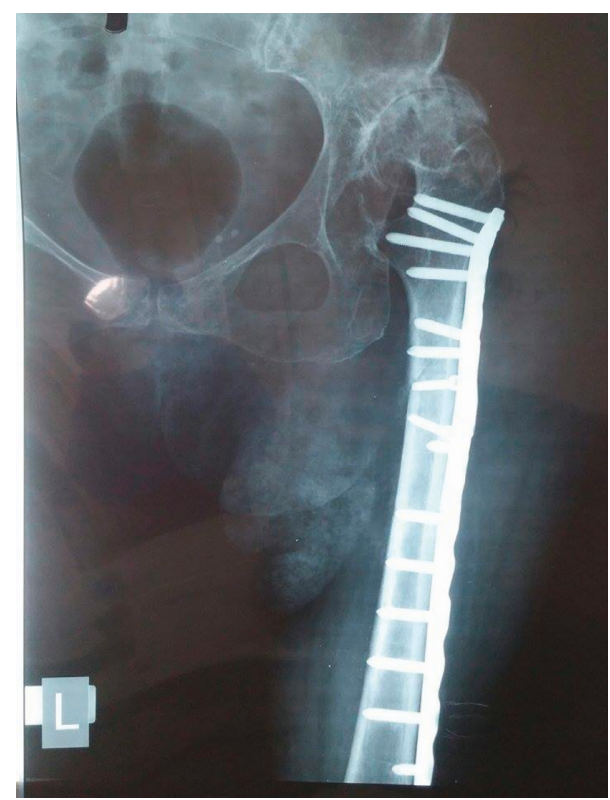

FIGURE 4: Anteroposterior radiograph of the left femur six weeks postoperatively.

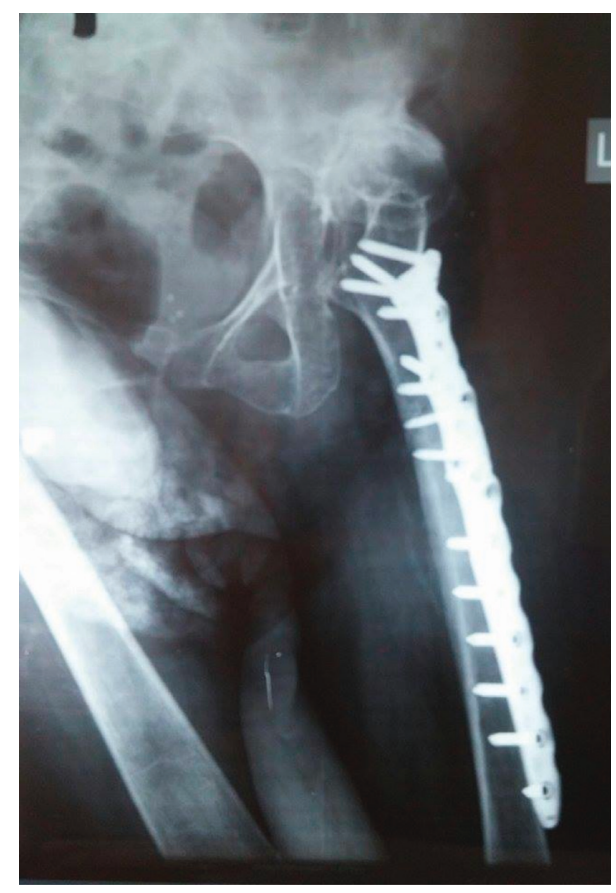

Figure 5: Profile radiograph of the left femur six weeks postoperatively.

great difficulties and potential intraoperative obstacles and risks [13]. The increased neck-shaft angle combined with the increased anteversion also exclude the fixation with dynamic hip screw (DHS) or other angled plates. Reconstruction with total hip arthroplasty (THA) with long femoral stem was an option thoroughly considered since that would confront the patient's both problems. Nonetheless, the modified anatomy that we have already mentioned makes THA a demanding and challenging technique with failure rates ranging from
$14 \%$ to $29 \%$ in ankylosed hip [13-15]. Tsakotos et al. successfully treated a midshaft femoral fracture in a 57-year-old lady with coexisting congenital dysplasia and ankylosis of the ipsilateral hip, by performing total hip arthroplasty with the use of a long stem that acted as an intramedullary nail [11]. However, in our case, the patient suffered from bilateral CHD, so THA would indicate a second operation in the future for the reconstruction of the contralateral hip in order to confront the consequent leg length discrepancy. Additionally, our patient was 74 years old, ambulatory with a walking stick, capable of all self-care, and contented with her previous walking status. Furthermore, the patient did not experience severe pain before the injury, neither nocturnal pain, and she was not receiving analgetics in a regular basis. The preexisting limp did not affect her everyday activities and her quality of life. As a result, the patient had never sought treatment before, and she had never been in a list for surgery. However, the option of a total hip arthroplasty with subtrochanteric shortening was discussed with her but was rejected as she did not accept the possible risks of such an operation.

By contrast, open internal fixation using this specially designed periprosthetic femur plate proved to be a successful method of stabilization after open anatomical reduction. The specific plate fits anatomically to the lateral wall of the femur, extends to the greater trochanter, and facilitates the placement of multiple bicortical locking screws of various diameter sizes in different directions proximally and distally, adding stability to the fixation.

\section{Consent}

Informed written consent was obtained prior to any diagnostic procedures or treatment and also prior to publication of case details.

\section{Conflicts of Interest}

The authors declare that they have no conflicts of interest regarding the publication of this paper.

\section{References}

[1] R. Loder and E. Skopelia, "The epidemiology and demographics of hip dysplasia," ISRN Orthopedics, vol. 2011, Article ID 238607, 46 pages, 2011.

[2] J. F. Crowe, V. J. Mani, and C. S. Ranawat, "Total hip replacement in congenital dislocation and dysplasia of the hip," Journal of Bone \& Joint Surgery, vol. 61, no. 1, pp. 15-23, 1979.

[3] G. Hartofilakidis, K. Stamos, and T. T. Ioannidis, "Low friction arthroplasty for old untreated congenital dislocation of the hip," Journal of Bone and Joint Surgery, vol. 70, no. 2, pp. 182-186, 1988.

[4] G. Hartofilakidis, C. K. Yiannakopoullos, and G. C. Babis, "The morphologic variations of low and high hip dislocation," Clinical Orthopaedics and Related Research, vol. 466, no. 4, pp. 820-824, 2008.

[5] A. B. Mullaji and R. C. Todd, "Late ipsilateral trochanteric fractures in patients with long-standing fusion of the hip," Injury, vol. 22, no. 3, pp. 233-235, 1991. 
[6] S. Asakawa, T. Mammoto, and A. Hirano, "Proximal femoral fracture in hip arthrodesis treated with double reconstruction plates," Case Reports in Orthopedics, vol. 2017, Article ID 5246080, 5 pages, 2017.

[7] A. Manzotti, N. Confalonieri, and C. Pullen, "Intertrochanteric fracture of an arthrodesed hip," Journal of Bone and Joint Surgery, vol. 89, no. 3, pp. 390-392, 2007.

[8] T. C. Wong and I. S. Rikhraj, "Femoral shaft fracture in a hip arthrodesis: two cases of retrograde interlocking nailing," Singapore Medical Journal, vol. 45, no. 2, pp. 85-87, 2004.

[9] F. M. Darwish and W. Haddad, "Intertrochanteric fracture under an arthrodesed hip," American Journal of Case Reports, vol. 14, pp. 150-152, 2013.

[10] A. P. Wulke, K. Mader, and D. Pennig, "Femoral neck fracture in an arthrodesed hip treated by a supracondylar intramedullary locked nail," Journal of Orthopaedic Trauma, vol. 18, no. 2, pp. 116-118, 2004.

[11] G. A. Tsakotos, S. D. Koutsostathis, and G. A. Macheras, "Treatment of a femoral shaft fracture in a patient with congenital hip disease: a case report," Journal of Medical Case Reports, vol. 4, no. 1, p. 221, 2010.

[12] W. M. Ricci, B. Gallagher, and G. J. Haidukewych, "Intramedullary nailing of femoral shaft fractures: current concepts," Journal of the American Academy of Orthopaedic Surgeons, vol. 17, no. 5, pp. 296-305, 2009.

[13] J. N. Argenson, X. Flecher, S. Parratte, and J. M. Aubaniac, "Anatomy of the dysplasia hip and consequences for total hip arthroplasty," Clinical Orthopaedics and Related Research, vol. 465, pp. 40-45, 2007.

[14] D. J. Kilgus, H. C. Amstutz, M. A. Wolgin, and F. J. Dorey, "Joint replacement for ankylosed hips," Journal of Bone and Joint Surgery Series A, vol. 72, no. 1, pp. 45-54, 1990.

[15] G. M. Strathy and R. H. Fitzgerald Jr., "Total hip arthroplasty in the ankylosed hip. A ten-year follow-up," Journal of Bone \& Joint Surgery, vol. 70, no. 7, pp. 963-966, 1988. 


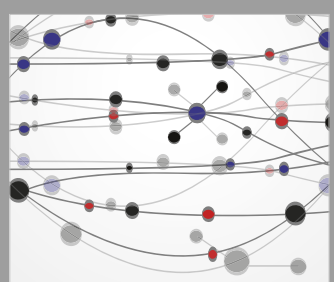

The Scientific World Journal
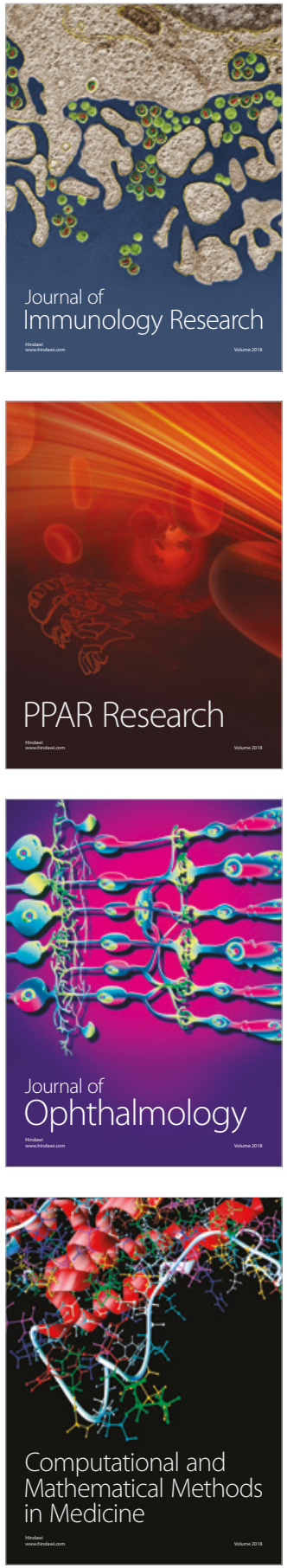

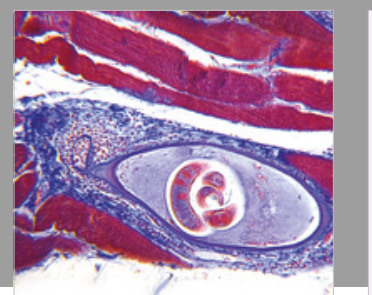

Gastroenterology Research and Practice

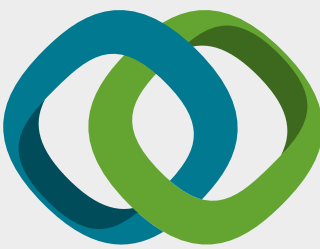

\section{Hindawi}

Submit your manuscripts at

www.hindawi.com
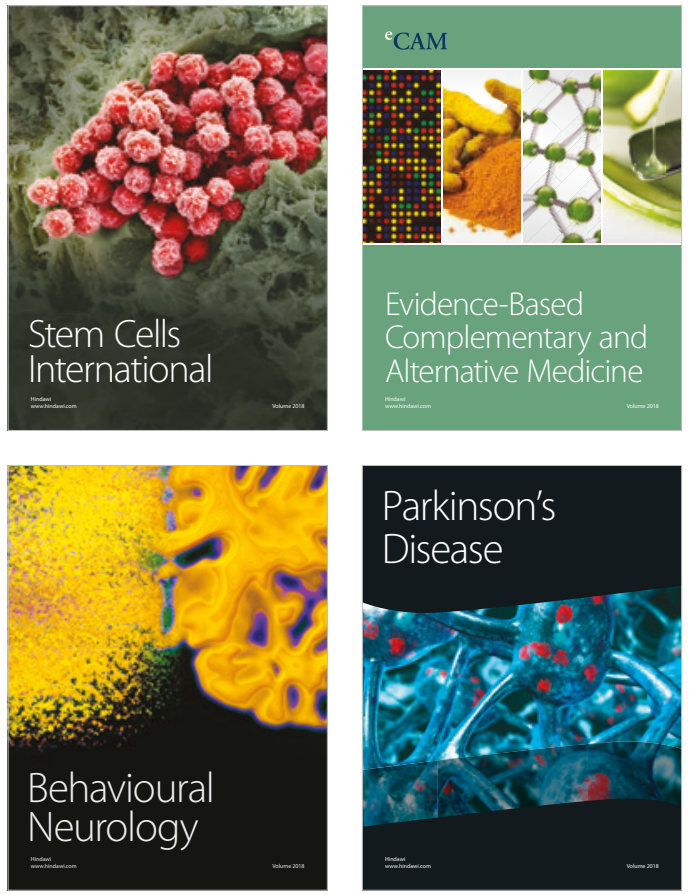

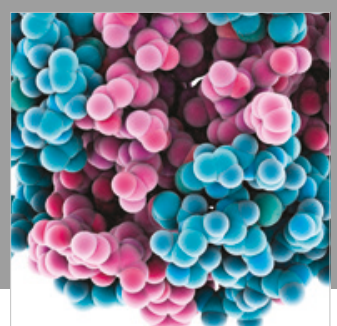

ournal of

Diabetes Research

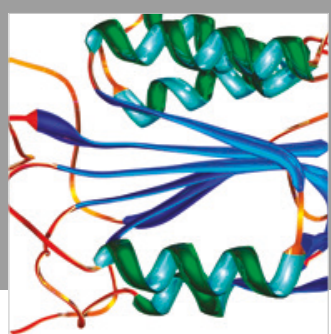

Disease Markers
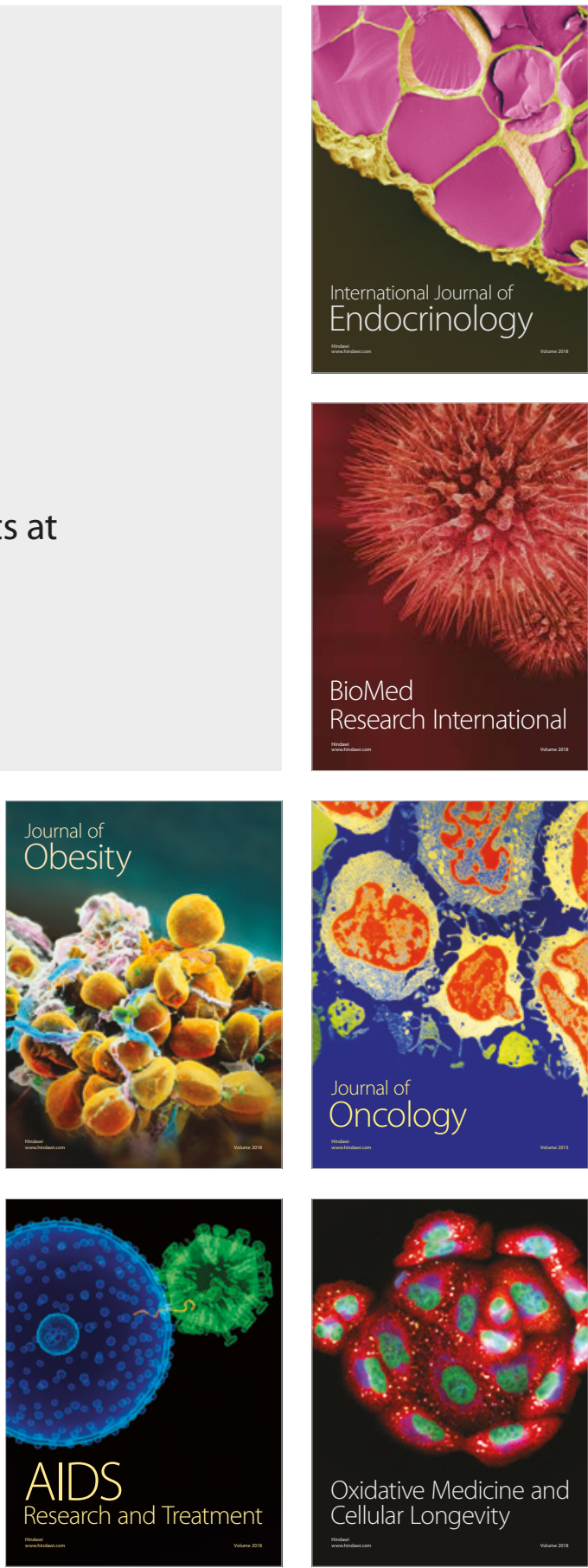